\section{Misunderstanding risk}

\section{To the editor:}

In his letter [Bio/Technology 13:1 142 (1995)], the U.S. Department of Agriculture's (USDA's) Alvin Young misunderstands - not for the first time - what constitutes bona fide risk-based policies. For example, he cites two attempts by USDA to craft biosafety guidelines for rDNA-manipulated organisms used in research involving field trials. Sadly, these scientifically flawed guidelines have been the major "accomplishments" of more than a decade of work by Young's Office of Agricultural Biotechnology $(\mathrm{OAB})$ and its Agricultural Biotechnology Research Advisory Committee (ABRAC). The first was an abortive four-year (1987-91) exercise during which the ABRAC acceded to the OAB's vision of inherent, unique biotechnology risks and need for extensive government oversight of research. Published for public comment in 1991, the final guidelines focused on the use of a single genetic technique. They placed OAB and USDA squarely in conflict with the conclusions and recommendations of national and international scientific groups that concurred with the U.S. National Academy of Sciences' 1987 white paper, which concluded that "[a]ssessment of the risks of introducing rDNA-engineered organisms into the environment should be based on the nature of the organisms and the environment into which the organism is introduced, not on the method by which it was produced."

The OAB approach is likewise inconsistent with official U.S. government policy, as described in the socalled "scope policy" [Federal Register 57:6753-6762 (1992)]. The ABRAC guidelines were rejected by senior science policymakers in the Bush administration, a sound policy decision that protected the research community from unnecessary USDA intervention and that spared taxpayers the need for increases in $\mathrm{OAB}$ manpower and budget. Young responded to this rejection by printing and mailing (at taxpayers' expense) thousands of copies of the guidelines, bound and presented in a way that implied that they were en route to official sanction. The second OAB effort cited by Young targeted recombinant DNA-modified aquatic organisms, with similar biosafety goals. Young notes in his letter that USDA has "found it useful to focus on groups of organisms with common biological traits and risk factors" in a recent USDA document, "Performance Standards for Safely Conducting Research with Genetically Modified Fish and Shellfish." Do rDNAmodified fish and shellfish possess "common biological traits and risk factors" not shared by fish and shellfish modified by other techniques? Of course not: That was an important corollary of the National Academy of Science and National Research Council (1989) reports. Unfortunately, Young's latest set of regulations-in-waiting illustrates once again a fundamental lack of understanding or unwillingness to adhere to the scientific principles underlying both risk analysis and the broad consensus that there is no evidence of unique risks conferred by the use of the newer molecular techniques. Putting the conundrum another way, if there is a need for additional regulation of new rDNAmodified varieties of fish and shellfish, there is likely also to be such a need for new organisms made using less precise techniques — or for wild-type organisms introduced into unfamiliar ecosystems ["exotics;" see, for example, Miller et al., Australasian Biotechnology 5:238-41 (1995)]. The very title of the more recent $\mathrm{OAB}$ document is revealing, in that it contains an oxymoron: Limiting the scope to genetically modified (rDNA-manipulated) organisms is fundamentally incompatible with genuine performance standards. Restricting the scope to rDNA-modified fish and shellfish represents regulation on the basis of an arbitrary and irrelevant fact, rather than on the basis of risk-related traits or characteristics. This approach imposes, in effect, the antithesis of a performance standard-a design standard. The scope of what is encompassed by the document is defined merely by genetic technique, not by a category enhanced in risk (such as pathogens or environmental nuisances) or by other performancerelated characteristics.

Young complains in his letter that our paper describing a risk-based algorithm for field trials [Miller et al., Bio/Technology 13:955-959 (1995)] neglected to mention the $\mathrm{OAB}$ effort. In fact, a previous publication that described an earlier version of our algorithm [Miller, H.I., R.H. Burris, A.K. Vidaver, and N.A. Wivel. Science 250:490 (1990)] did note the contributions of USDA. However, given the significant scientific shortcomings and obsolescence of the OAB approach (which was never, to the best of our knowledge, published in any scientific or public policy journal), further acknowledgment was unwarranted and irrelevant to our paper. Young refers repeatedly in his letter to the prodigious labors and myriad participants in USDA's various efforts to draft guidelines. In two important respects, however, the formulation of public policy resembles laboratory research: (1) Employing valid basic assumptions is essential to a correct result; and (2) the quality of the outcome may not be proportional to the number of human-hours expended. Young's focus on using invalid assumptions corrupts the product, wastes the efforts of USDA's staff and extramural advisors, trivializes the role of legitimate government regulation, and makes for flawed public policy.

Henry I. Miller

Hoover Institution \& Institute for International Studies

Stanford University Stanford, CA 94305-6010

Susanne L. Huttner

University of California Systemwide Research \& Education Program University of California, Berkeley, CA 94920-3100

David W. Altman Nashville, TN 81837-4030
Comments and opinions on editorials, articles, and research in $\mathrm{Bio} / \mathrm{Technology}$ are welcome. Letters to the Editor may be addressed to: 345 Park Avenue South,

New York, $N Y$

10010 ,

fax: 212 696-9635, (e-mail: s.hassler @natureny.com). 